\title{
ESTOQUE DE SERAPILHEIRA EM FLORESTA EM RESTAURAÇÃO E EM FLORESTA ATLÂNTICA DE TABULEIRO NO SUDESTE BRASILEIRO' ${ }^{1}$
}

\author{
Geanna Gonçalves de Souza Correia ${ }^{2}$, Sebastião Venâncio Martins ${ }^{3 *}$, Aurino Miranda Neto ${ }^{4}$ Kelly de \\ Almeida Silva ${ }^{4}$

\footnotetext{
${ }^{1}$ Recebido em 07.02.2014 aceito para publicação em 11.11.2015.

${ }^{2}$ Universidade Federal de Viçosa, Programa de Pós-Graduação em Botânica, Viçosa, MG - Brasil. E-mail: <geannagsc@hotmail.com>. ${ }^{3}$ Universidade Federal de Viçosa, Centro de Ciências Agrárias, Departamento de Engenharia Florestal, Vicosa, MG - Brasil. E-mail:<venancio@ufv.br>.

${ }^{4}$ Universidade Federal de Viçosa, Programa de Pós-Graduação em Ciência Florestal, Viçosa, MG - Brasil. E-mail:<aur.neto@gmail.com>.

*Autor para correspondência.
}

\begin{abstract}
RESUMO - Neste trabalho, objetivou-se quantificar e comparar a serapilheira acumulada em uma floresta em restauração (FR) há 23 anos com uma Floresta Ombrófila Densa primária (FP) adjacente. Foram alocadas 30 parcelas permanentes de $3 \times 3 \mathrm{~m}$ em cada área. No centro de cada parcela foi coletada a serapilheira $(0,50 \times 0,50 \times 0,05 \mathrm{~m})$. O material foi seco em estufa a $70{ }^{\circ} \mathrm{C}$, durante $48 \mathrm{~h}$, e mensurada a massa de matéria seca. Foi calculada a matriz de correlação de Pearson entre os valores de serapilheira acumulada e as variáveis ambientais índice de área de planta (IAP), compactação do solo e densidade de indivíduos arbustivo-arbóreos do estrato de regeneração natural. Os valores médios encontrados (FR $=3.177 \pm 937 \mathrm{~kg}$.ha- $1 ; \mathrm{FP}=4.411 \pm 1.389 \mathrm{~kg}$.ha-1) de serapilheira acumulada apresentaram diferença significativa entre as duas áreas estudadas $(\mathrm{p}<0,001)$. A serapilheira acumulada apresentou correlação significativa $(p<0,001)$ com a compactação do solo e índice de área de planta.
\end{abstract}

Palavras-chave: Indicadores de Restauração; Restauração Florestal; Floresta de Referência.

\section{STOCK OF LITTER IN RESTORATION FOREST AND TABLELAND ATLANTIC FOREST IN SOUTHEASTERN BRAZIL}

\begin{abstract}
The objective of this study was to quantify and compare the accumulated litter at a forest restoration (FR) for 23 years with a primary Tropical Rain Forest (FP). Thirty permanent plots of 3 $\times 3 \mathrm{~m}$ were placed in each area. In the center of each plot the litter $(0,50 \times 0,50 \times 0,05 \mathrm{~m})$ was collected. The material was dried at $70^{\circ} \mathrm{C}$ for 48 hours and the dry mass was measured. The matrix of Pearson correlation between the values of accumulated litter and environmental variables plant area index (PAI), soil compaction and density of shrub and tree individuals of natural regeneration layer were calculated. The mean values found ( $\left.F R=3,177 \pm 937 \mathrm{~kg} . \mathrm{ha}^{-1} ; F P=4,411 \pm 1,389 \mathrm{~kg} . \mathrm{ha}^{-1}\right)$ of accumulated litter showed significant difference between the two study areas $(p<0.001)$. The accumulated litter showed significant correlation $(p<0.001)$ with soil compaction and plant area index.
\end{abstract}

Keywords: Indicators of Restoration; Forest Restoration; Reference Forest. 


\section{INTRODUÇÃO}

Todos os ecossistemas, atualmente, possuem um histórico processo de alteração de suas áreas originais decorrente de diferentes ações antrópicas. Esse processo ocasiona a perda da cobertura vegetal e acarreta grande fragmentação de hábitats e consequente impactos na biodiversidade, além de potenciais perdas de processos biológicos e serviços ecossistêmicos. Em contrapartida, como forma de reverter esse cenário, encontra-se a restauração florestal. Representada por um conjunto de modelos e técnicas, a restauração florestal se aprimorou ao longo dos últimos anos e "visa restaurar funções dos ecossistemas e recuperar muitos componentes da biodiversidade original" (CHAZDON, 2008).

Quando os plantios de espécies florestais com fins de restauração florestal são realizados em áreas contíguas a remanescentes naturais, apresentam-se de forma ainda mais eficientes em comparação com pastagens e campos agrícolas (DENYER et al., 2006). Esse fato é observado em muitos estudos, que constataram que esses ambientes em processo de restauração diminuem os efeitos de borda e diversas pressões ambientais nos fragmentos naturais (DENYER et al., 2006).

Através de técnicas de restauração, dá-se início ao processo de sucessão ecológica nas áreas alteradas e degradadas, subsidiando a formação de um ambiente com condições de autossuficiência após anos de implantação e monitoramento/manutenção daquele novo ambiente. Como forma de monitorar esses ambientes em restauração, quanto ao seu sucesso, são aplicados diferentes indicadores, como a regeneração natural, banco e chuva de sementes, acúmulo de serapilheira, entre outros (RODRIGUES; GANDOLFI, 1998; MARTINS; KUNZ, 2007; MARTINS, 2009; BRANCALION et al., 2012).

Entre esses indicadores de avaliação e monitoramento da restauração florestal, um dos mais importantes e de mais rápida e precisa determinação é a serapilheira depositada na superfície do solo, uma vez que a serapilheira é considerada a principal forma de adição de matéria orgânica e nutrientes ao solo da floresta (POGGIANI et al., 1998), através do sistema solo-plantaserapilheira. Esse sistema é importante, principalmente, em ambientes com solos altamente intemperizados e

Revista Árvore, Viçosa-MG, v.40, n.1, p.13-20, 2016 com poucos nutrientes disponíveis, encontrado em grande parte das florestas tropicais (POGGIANI, 2012).

A serapilheira formada por material de origem vegetal e animal pode apresentar variação na sua deposição no solo em função de fatores como o clima, o estádio sucessional da vegetação e a fertilidade do solo (VITOUSEK; SANFORD, 1986). Assim, o estoque de serapilheira acumulada também sofre variação em decorrência do estágio sucessional e do ecossistema, podendo ser inferior ou superior à produzida no ano, em virtude da sua taxa de decomposição no referido ambiente (OLSON, 1963).

Nesse contexto, este estudo visou quantificar e comparar a serapilheira acumulada em uma área em processo de restauração há 23 anos com uma área de Floresta Ombrófila Densa primária adjacente, bem como relacionar o estoque de serapilheira com índice de área de planta e a compactação do solo de ambas as áreas.

\section{MATERIAL E MÉTODOS}

\subsection{Caracterização da área de estudo}

A pesquisa foi realizada na Reserva Natural Vale (RNV), localizada nos Municípios de Linhares e Jaguaré, Norte do Estado do Espírito Santo (1906'-19¹8'S e $39^{\circ} 45^{\prime}-40^{\circ} 19^{\prime} \mathrm{W}$ ) (Figura 1). Com cerca de 22.000 ha, essa reserva, juntamente com a Reserva Biológica de Sooretama (sob gestão do Instituto Chico Mendes de Conservação da Biodiversidade - ICMBio), contemplam uma área de aproximadamente 46.000 ha, representando o maior maciço florestal do Estado. A vegetação é classificada por Veloso et al. (1991) como Floresta Ombrófila Densa de Terras Baixas, sendo também conhecida como Floresta de Tabuleiro. O clima na classificação de Köppen é do tipo tropical quente e úmido, com estação chuvosa no verão e seca no inverno, com precipitação pluviométrica média anual de 1.202 mm e a temperatura média de $23,3^{\circ} \mathrm{C}$ (JESUS; ROLIM, 2005). Essa temperatura se encontra sob os Tabuleiros Terciários do Grupo Barreiras e apresenta relevo relativamente plano com altitudes entre 28 e $65 \mathrm{~m}$, constituído, principalmente, por Argissolo Amarelo (JESUS; ROLIM, 2005).

O estudo foi desenvolvido em duas áreas adjacentes: uma área constituída por Floresta Ombrófila Densa primária (FP) e outra por Floresta em Restauração (FR). A FR possui 7,8 ha e a sua implantação foi em novembro/ 1989, com o plantio em linha de 58 espécies, 


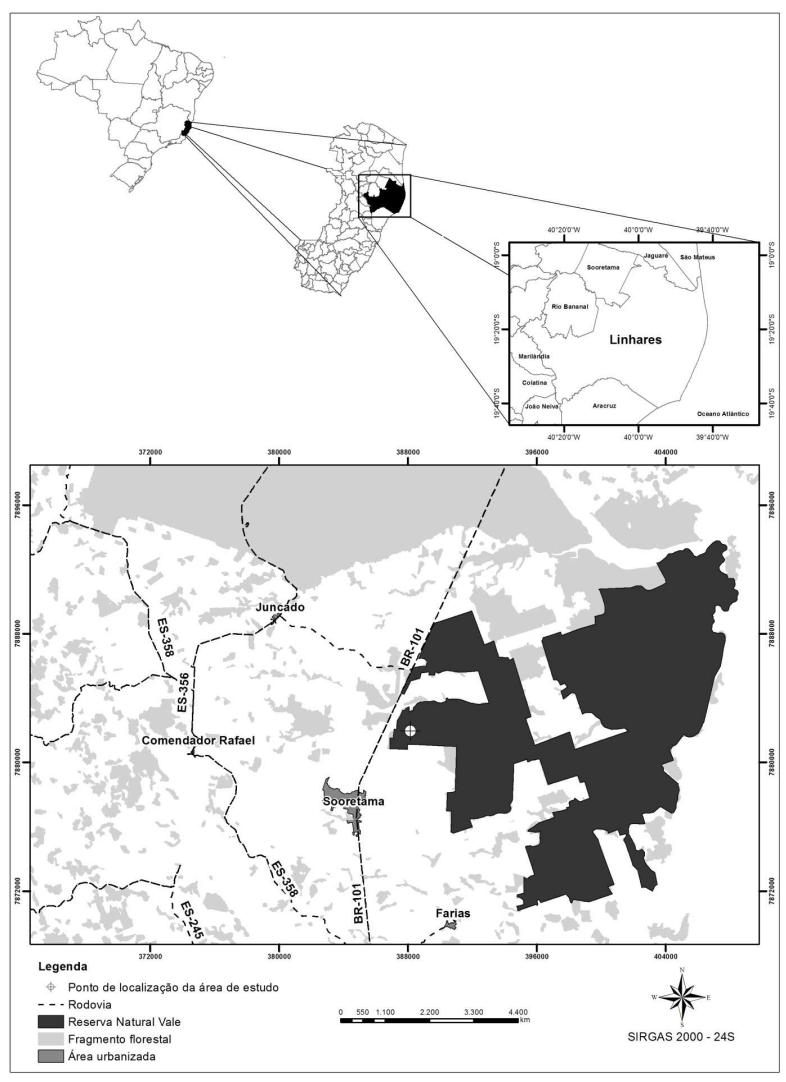

Figura 1 - Área de estudo em Floresta de Tabuleiro no Espírito Santo, Região Sudeste do Brasil.

Figure 1 - Study area in Tableland Forest in Espirito Santo, southeastern Brazil.

compreendidas, em sua maioria, por nativas. No início da implantação, a área encontrava-se com regeneração de Eucalyptus spp. e infestada por capim-colonião (Panicum maximum Jacq.) e sapê (Imperata brasiliensis Trin.). Como operações silviculturais, foram realizadas a eliminação da regeneração do eucalipto e a roçada manual total, no espaçamento de 2 × 2 m, e adubação com $200 \mathrm{~g}$ de superfosfato simples por cova. Fez-se, ainda, o controle das formigas-cortadeiras com a utilização de isca granulada à razão de $10 \mathrm{~g} / \mathrm{m}^{2}$ de formigueiro (informações cedidas pela Vale $\mathrm{S} / \mathrm{A}$ ).

\subsection{Coleta e análise dos dados}

Foram alocadas de forma sistemática 30 parcelas permanentes de $3 \times 3 \mathrm{~m}$ em cada área, totalizando 60 parcelas $\left(540 \mathrm{~m}^{2}\right)$, distanciadas em $5 \mathrm{~m}$ uma das outras (Figura 2).

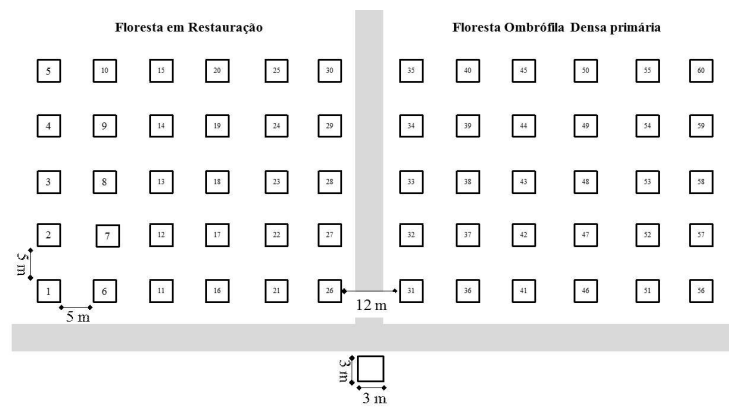

Figura 2 - Esquema da distribuição das parcelas na Reserva Natural Vale, Linhares, ES. Em cinza = vias de acesso.

Figure 2-Distribution of plots in Reserva Natural Vale, Linhares, ES. Gray = access roads.

No centro de cada parcela, foi alocado um gabarito de madeira de $50 \times 50 \mathrm{~cm}\left(0,25 \mathrm{~m}^{2}\right)$, no qual foi coletado todo o material orgânico não decomposto (folhas, ramos, frutos e flores) contido no interior. Em seguida, esse foi embalado em sacos de papel e transferido para o Laboratório de Restauração Florestal (LARF) da Universidade Federal de Viçosa (UFV), onde foi seco em estufa a $70{ }^{\circ} \mathrm{C}$, durante $48 \mathrm{~h}$, e posteriormente mensuradas as massas de matéria seca em balança analítica de precisão.

A quantidade de serapilheira acumulada encontrada no gabarito de madeira $\left(\mathrm{g} / 0,25 \mathrm{~m}^{2}\right)$ foi estimada para $\mathrm{kg} \cdot \mathrm{ha}^{-1}$ e as médias, obtidas na Floresta em Restauração e comparadas com a Floresta Ombrófila Densa através do teste $\mathrm{t}$, de Student, para amostras independentes com o auxílio do software STATISTICA 7.0 (STATSOFT, 2004).

Para avaliar a relação da serapilheira com outras variáveis, foi calculada a matriz de correlação de Pearson entre os valores de serapilheira acumulada, índice de área de planta (IAP), compactação do solo e densidade de indivíduos arbustivo-arbóreos do estrato de regeneração natural.

O Índice de Área de Planta (IAP) representa a área ocupada pelos elementos do dossel (folhas, ramos, flores, frutos) em relação à área do solo (BRÉDA, 2003). Para obtenção do IAP, foi tirada uma fotografia hemisférica digital no centro de cada parcela, com o equipamento CI-1 10 Digital Plant Canopy Imager $\AA$, e em seguida as fotografias foram processadas no Programa Gap Light Analyzer 2.0 (FRAZER et al., 1999).

Revista Árvore, Viçosa-MG, v.40, n.1, p.13-20, 2016

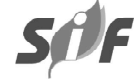


A compactação do solo foi mensurada até 10 $\mathrm{cm}$ de profundidade utilizando um penetrômetro digital de impacto modelo penetrolog PLG1020 Falker®. Para as análises, foi utilizado o valor mais elevado da pressão atingido entre 0 e $10 \mathrm{~cm}$.

O número de indivíduos do estrato de regeneração foi obtido através da contabilização das árvores e arbustos com altura $\geq 0,30 \mathrm{~m}$ e circunferência à altura do peito $(\mathrm{CAP}=1,30 \mathrm{~m}$ do solo $) \leq 15,0 \mathrm{~cm}$ presentes nas parcelas.

\section{RESULTADOS}

Os valores médios encontrados $(\mathrm{FR}=3.177 \pm 937$ kg.ha-1 FP $\left.=4.411 \pm 1.389 \mathrm{~kg} \cdot \mathrm{ha}^{-1}\right)$ de serapilheira acumulada apresentaram diferença significativa entre as duas áreas estudadas $(P<0,001)$.

Seguindo a mesma tendência, os valores encontrados para o IAP $(\mathrm{FR}=1,8 \pm 1,1 ; \mathrm{FP}=4,8 \pm 1,5)$ e a compactação do solo $(\mathrm{FR}=1.481,8 \pm 573,1 \mathrm{kPa} ; \mathrm{FP}=552,2 \pm 433,4$ $\mathrm{kPa})$ também apresentaram diferenças significativas $(P<0,001)$. O número médio de indivíduos por parcela não apresentou diferença significativa $(P=0,66)(\mathrm{FR}$ $=11,6 \pm 6,9$ indivíduos; e FP $=12,3 \pm 5,9$ indivíduos).

A serapilheira acumulada apresentou correlação significativa $(P<0,01)$ positiva com o índice de área de planta (IAP) e negativa com a compactação do solo (Tabela 1 e Figura 3). Isso indica maior estoque de serapilheira nos locais com menor abertura de dossel e menor compactação do solo. Também houve correlação significativa negativa $(\mathrm{p}<0,01)$ entre IAP e compactação do solo (Tabela 1).

\section{DISCUSSÃO}

Os valores de serapilheira acumulada indicam que, mesmo após 23 anos de plantio, a área em restauração ainda não atingiu valores de estoque de serapilheira no solo semelhantes ao de uma Floresta de Tabuleiro em estágio avançado de sucessão. Cabe destacar que, embora tenha sido realizada a coleta apenas no verão, esses valores representam bem o estoque de serapilheira das duas florestas, uma vez que nas florestas ombrófilas as condições climáticas e a resposta da vegetação são menos variadas ao longo do ano, diferentemente do que ocorre nas florestas estacionais onde há maior queda do folhedo nas estações menos chuvosas e mais frias (MEGURO et al., 1979; MARTINS; RODRIGUES, 1999; BOREM; RAMOS, 2002; SILVA, 2012).

Esses valores obtidos tanto na FR quanto na FP se enquadram próximos ao limite inferior da faixa descrita por O'Connell e Sankaran (1997), os quais relataram que em florestas tropicais da América do Sul a quantidade de serapilheira acumulada varia entre 3.100 e 16.500 kg.ha ${ }^{-1}$. Esses resultados também corroboram com os do estudo de Kindel et al. (1999), também realizado nas Florestas de Tabuleiro da RNV, com valor médio de $4.000 \mathrm{~kg} . \mathrm{ha}^{-1}$ entre as estações de verão e inverno. Esses autores concluíram que o material orgânico presente nesse ambiente apresenta elevada taxa de decomposição, deixando alguns locais desnudos durante o ano. Fato esse que pode justificar os baixos valores encontrados em comparação com outros estudos em florestas no Espírito Santo (ANDRADE et al., 2003; GONÇALVES, 2008; GODINHO, 2011; KLIPPEL, 2011; SPERANDIO et al., 2012; SPERANDIO, 2013).

Em outra área em restauração, há apenas três anos na mesma reserva o valor médio de serapilheira acumulada foi muito superior $\left(7.400 \mathrm{~kg} \cdot \mathrm{ha}^{-1}\right)$ em razão, provavelmente, do fato de esse ambiente ser mais jovem e possuir menor taxa de decomposição da serapilheira (KLIPPEL, 2011).

A correlação positiva entre a serapilheira acumulada e o IAP indica que, quanto maior o IAP, maior a concentração de serapilheira acumulada sobre o solo, considerando o conjunto de parcelas das duas áreas

Tabela 1 - Correlação de Pearson entre serapilheira acumulada, compactação do solo, índice de área de planta (IAP) e número de indivíduos da regeneração (NI) para o conjunto de 60 parcelas das duas florestas avaliadas, Reserva Natural Vale, Linhares, ES.

Table 1 - Pearson correlation among accumulated litter, soil compaction, plant area index (PAI) and number of individuals of regeneration (NI) for the 60 plots of the two evaluated forests, Reserva Natural Vale, Linhares, ES

\begin{tabular}{|c|c|c|c|c|}
\hline & Serapilheira & Comp. do solo & IAP & $\mathrm{NI}$ \\
\hline Serapilheira & 1,000 & - & - & - \\
\hline Comp. do solo & $-0,397^{*}$ & 1,000 & - & - \\
\hline IAP & $0,473 *$ & $-0,520 *$ & 1,000 & - \\
\hline NI & 0,019 & $-0,002$ & 0,120 & 1,000 \\
\hline
\end{tabular}

Revista Árvore, Viçosa-MG, v.40, n.1, p.13-20, 2016 

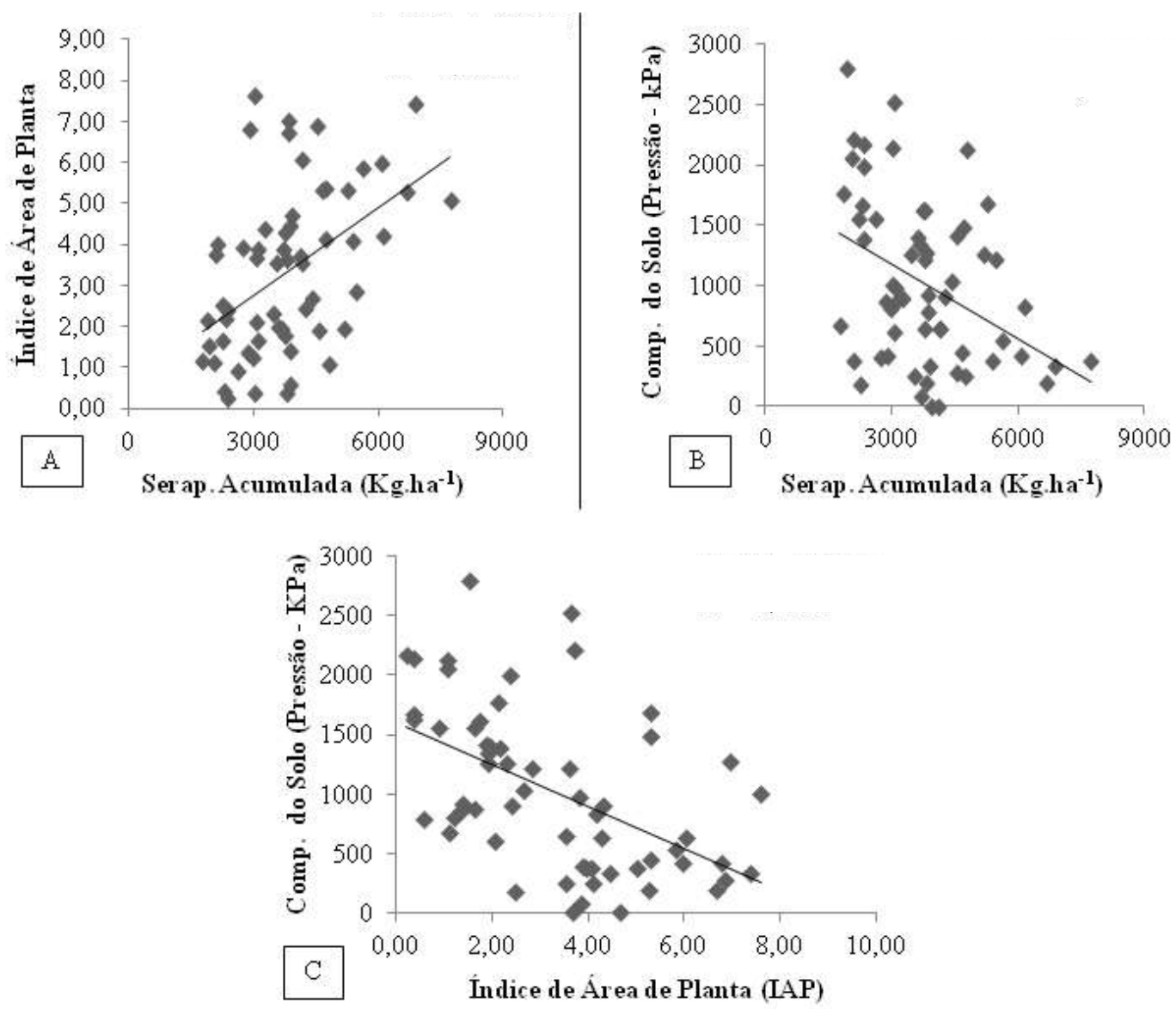

Figura 3 - Correlação entre a serapilheira acumulada e IAP (A) entre serapilheira acumulada e compactação do solo (B) e entre o IAP e a compactação do solo (C), para o conjunto de 60 parcelas das duas florestas avaliadas, Reserva Natural Vale, Linhares, ES.

Figure 3 - Correlation between accumulated litter and PAI $(A)$, between accumulated litter and soil compaction $(B)$ and between PAI and soil compaction (C), for the 60 plots of the two evaluated forests, Reserva Natural Vale, Linhares, ES.

(FR e FP). Essa relação se explica pelo fato de o IAP refletir o nível de cobertura do dossel florestal, ou seja, sob um dossel mais fechado a deposição de serapilheira tende a ser maior que em um dossel mais aberto.

A correlação negativa entre a serapilheira acumulada e a compactação do solo mostra que a serapilheira apresentou maiores concentrações em solos menos compactados, considerando o conjunto de parcelas das duas áreas. As diferenças de compactação do solo entre as duas áreas se devem aos seus diferentes históricos de uso, já que a floresta primária não sofreu qualquer tipo de intervenção antrópica que gere compactação de solo, e a área da floresta em restauração foi ocupada por reflorestamento de eucalipto no passado, tendo sido submetida a níveis de compactação do solo nas operações de colheita de madeira.

A correlação negativa entre o IAP e a compactação do solo evidencia que em locais com dossel mais fechado o nível de compactação do solo é menor em razão, possivelmente, do favorecimento de um ambiente mais úmido. Já que um dossel mais fechado aumentará o estoque de serapilheira no solo, assim, segundo Sperandio et al. (2012), ele pode minimizar o impacto

Revista Árvore, Viçosa-MG, v.40, n.1, p.13-20, 2016 
das gotas de chuva, diminuir a temperatura do solo e aumentar a ciclagem de nutrientes. Portanto, esses fatores podem favorecer o solo a apresentar nível menor de compactação.

Santos e Válio (2002) observaram, no Estado de São Paulo, que a remoção da serapilheira no interior da mata aumentou a emergência de plântulas. Já neste estudo o número de indivíduos amostrados por parcela não apresentou nenhuma correlação com a quantidade de serapilheira acumulada no solo. A maior produção de serapilheira não está condicionada à densidade de indivíduos da regeneração. De acordo com Songwe et al. (1988) e Werneck et al. (2001), a quantidade de serapilheira relaciona-se mais à estrutura da floresta, principalmente no desenvolvimento do dossel.

\section{CONCLUSÃO}

Os resultados indicaram que a floresta em processo de restauração apresenta estoque de serapilheira inferior a um trecho de Floresta Ombrófila Densa primária adjacente.

O estoque de serapilheira é maior nos locais com menor abertura de dossel e menor compactação do solo.

\section{AGRADECIMENTOS}

À CAPES, pela bolsa de Mestrado à primeira autora; ao CNPq, pela bolsa de Produtividade em Pesquisa ao segundo autor; à Vale, pelo apoio ao projeto; e, em especial, ao G. Terra, pelo apoio prestado durante a pesquisa. Também a A. Christo, B. Siqueira, C. Nogueira, M. V. Campos e T. Diniz, pelo apoio nas atividades de campo e de laboratório.

\section{REFERÊNCIAS}

ANDRADE, F.N.; PEREZ, D.V.; GARAY, I. Heterogeneidade interna de fragmentos florestais: o impacto antrópico sobre as características do solo em remanescentes de Floresta Atlântica de Tabuleiros, Sooretama, ES. In: CONGRESSO DE ECOLOGIA DO BRASIL, 7., Caxambu, 2003. Anais... Caxambu: SEB, 2003. 2p.

BOREM, R.A.; RAMOS, D.P. Variação estacional e topográfica de nutrientes na serapilheira de um

Revista Árvore, Viçosa-MG, v.40, n.1, p.13-20, 2016 fragmento de mata atlântica. Cerne, v.8, n.2, p.42-59, 2002.

BRANCALION, P.H.S.; VIANI, R.A.G.; RODRIGUES, R.R.; GANDOLFI, S. Avaliação e monitoramento de áreas em processo de restauração. In: MARTINS, S.V. (Ed.).

Restauração ecológica de ecossistemas degradados. Viçosa, MG: Universidade Federal de Viçosa, 2012. p.262-293.

BRÉDA, N.J.J. Ground-based measurements of leaf area index: a review of methods, instruments and current controversies.

Journal of Experimental Botany, v. 54, n.392, p.2403-2417, 2003.

CHAZDON, R.L. Beyond deforestation: restoring forests and ecosystem services on degraded lands. Science, v.320, p.1458-1460, 2008.

DENYER, K.; BURNS, B.; OGDEN, J. Buffering of native forest edge microclimate by adjoining tree plantations. Austral Ecology, v.31, p.478-489, 2006.

FRAZER, G.W.; CANHAM, C.D.; LERTZMAN, K.P. Gap Light Analyzer (GLA):

Imaging software to extract canopy structure and gap light transmission indices from truecolour fisheye photographs, users manual and program documentation. New York: Simon Fraser University, Burnaby, British Columbia, and the Institute of Ecosystem Studies, 1999.

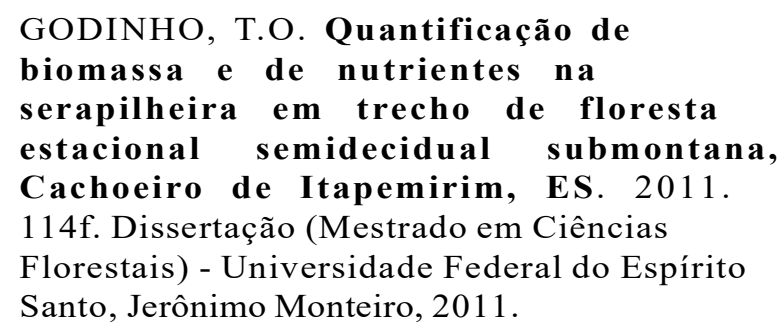

GONÇALVES, M.A.M. Avaliação da serapilheira em fragmento de floresta atlântica no sul do estado do Espírito Santo. 2008. 83f. Dissertação (Mestrado em Ciências Florestais) - Universidade Federal do Espírito Santo, Alegre, 2008. 
JESUS, R.M.; ROLIM, S.G. Fitossociologia da Mata Atlântica de Tabuleiro. Boletim Técnico da Sociedade de Investigações Florestais, v.19, p.1-149, 2005.

KINDEL, A.; BARBOSA, P.M.S.; PÉREZ, D.V.; GARAY, I. Efeito do extrativismo seletivo de espécies arbóreas da Floresta Atlântica de Tabuleiros na matéria orgânica e outros atributos do solo. Revista Brasileira de Ciência do Solo, v.23, n.3, p.465-474, 1999.

KLIPPEL, V.H. Avaliação de métodos de restauração florestal de Mata Atlântica de Tabuleiros. 2011. Dissertação (Mestrado em Ciências Florestais) - Universidade Federal do Espírito Santo, Alegre, 2011.

MARTINS, S.V.; RODRIGUES, R.R. Produção de serapilheira em clareiras de uma floresta estacional semidecidual no município de Campinas, SP. Revista Brasileira de Botânica, v.22, n.3, p.405-412, 1999.

MARTINS, S.V. Recuperação de áreas degradadas: ações em áreas de preservação permanente, voçorocas, taludes rodoviários e de mineração. 3. ed. Viçosa, MG: Aprenda Fácil, 2013. 264p.

MARTINS, S.V. Soil seed bank as indicator of forest regeneration potential in canopy gaps of a semideciduous forest in Southeastern Brazil. In: FOURNIER, M.V. (Ed.) Forest regeneration: ecology, management and economics. New York: Nova Science Publishers, 2009. p.113-128.

MARTINS, S.V.; KUNZ, S.H. Use of evaluation and monitoring indicators in a riparian forest restoration project in Viçosa, Southeastern Brazil. In: RODRIGUES, R.R.; MARTINS, S.V.; GANDOLFI, S. (Ed.) High diversity forest restoration in degraded areas: methods and projects in Brazil. New York: Nova Science Publishers, 2007. p.261-273.

MEGURO, M.; VINUEZA, G.N.; DELITTI, W.B.C. Ciclagem de nutrientes minerais na mata mesófila secundária - São Paulo. I - Produção e conteúdo de nutrientes minerais no folhedo. Boletim de Botânica, v. 7, p.11-31, 1979.

O'CONNELL, A.M.; SANKARAN, K.V. Organic matter accretion, decomposition and mineralisation. In: NAMBIAR, E.K.S.; BROWN, A.G. (Ed.). Management of soil, nutrients and water in tropical plantations forests. Canberra: ACIAR Australia/CSIRO, 1997. p.443-480.

OLSON, J. S. Energy storage and the balance of producers and decomposers in ecological systems. Ecology, v.44, p.322-331, 1963.

POGGIANI, F. Ciclagem de nutrientes em florestas do Brasil. In: MARTINS, S.V. (Ed.). Ecologia de florestas tropicais do Brasil. 2.ed. Viçosa, MG: Universidade Federal de Viçosa, 2012. p.175-251.

POGGIANI, F., STAPE, J.L., GONÇALVES, J.L.M. Indicadores de sustentabilidade das plantações florestais. Série Técnica IPEF, v.12, n.31, p.33-44, 1998.

RODRIGUES, R.R.; GANDOLFI, S. Restauração de florestas tropicais: subsídios para uma definição de metodologia e indicadores de avaliação e monitoramento. In: DIAS, L.E.; MELLO, J.W.V. (Ed.). Recuperação de áreas degradadas. Viçosa, MG: UFV/SOBRADE, 1988. p.203-215.

SANTOS, S.L.; VÁLIO, I.F.M. Litter accumulation and its effect on seedling recruitment in a Southeast Brazilian Tropical Forest. Revista Brasileira de Botânica, v.25, n. 1, p.89-92, 2002.

SILVA, R.D. Indicadores de recuperação ambiental em diferentes coberturas florestais, Alegre - ES. 2012. $61 \mathrm{f}$. Dissertação (Mestrado em Ciências Florestais) Universidade Federal do Espírito Santo, 2012.

SONGWE, N.C.; FASEHUN, F.E.; OKALI, D.U.U. Litterfall and productivity in a tropical rain forest, Southern Bankundu Forest, Camerron. Journal of Tropical Ecology, v.4, n.1, p. 25-37, 1988.

SPERANDIO, H.V. Tephrosia candida D.C. e Mimosa velloziana Mart: biomassa, serapilheira e fertilidade do solo. 2013.61f. Dissertação (Mestrado em Ciências Florestais) Universidade Federal do Espírito Santo, Jerônimo Monteiro, 2013.

Revista Árvore, Viçosa-MG, v.40, n.1, p.13-20, 2016 
SPERANDIO, H.V.; CECÍLIO, R.A.; SILVA, V.H.; LEAL, G.F.; BRINATE, I.B.; CALDEIRA, M.V.W. Emprego da serapilheira acumulada na avaliação de sistemas de restauração florestal em Alegre-ES. Floresta e Ambiente, v. 19, n.4, p.460-467, 2012.

STATSOFT, INC. Statistica - Data Analysis software system. Version 7.0.61.0. Tulsa: 2004.

VELLOSO, H.P.; RANGEL-FILHO, A.L.; LIMA, J.C.A. Classificação da vegetação brasileira, adaptada a um sistema universal. Rio de Janeiro: IBGE -
Departamento de Recursos Naturais e Estudos Ambientais, 1991.

VITOUSEK, P.M.; SANFORD, R.L. Nutrient cycling in moist tropical forest. Annual Review of Ecology and Systematics, v.17, p.137-167, 1986.

WERNECK, M.S.; PEDRALLI, G.; GIESEKE, L.F. Produção de serapilheira em trechos de uma floresta semidecídua com diferentes graus de perturbação na Estação Ecológica do Tripuí, Ouro Preto, MG. Revista Brasileira de Botânica, v.24, n.1, p.195-198, 2001. 